\title{
Photodegradation of Melamine Using Magnetic Silicon Quantum Dots
}

\author{
Iram MAHMOOD ${ }^{1 *}$, Ishfaq AHMAD ${ }^{2,3}$, Ishaq AHMAD ${ }^{4,5}$, Ting-kai ZHAO $^{5,6}$ \\ ${ }^{1}$ Department of Industrial Engineering, College of Engineering, King Khalid University, Abha 61413, Kingdom of Saudi \\ Arabia \\ ${ }^{2}$ Department of Mathematics, College of Science, King Khalid University, Abha 61413, Kingdom of Saudi Arabia \\ ${ }^{3}$ Department of Mathematics and Statistics, International Islamic University, Islamabad Pakistan \\ ${ }^{4}$ National Center for Physics, Islamabad, 44000 Pakistan \\ ${ }^{5}$ NPU-NCP Joint International Research Center for Advanced Nanomaterials and Defects Engineering, Northwestern \\ Polytechnical University, Xi'an 710072, China \\ ${ }^{6}$ School of Materials Science \& Engineering, Northwestern Polytechnical University, Xi'an 710072, China
}

crossref http://dx.doi.org/10.5755/j02.ms.22688

Received 04 February 2019; accepted 02 October 2019

\begin{abstract}
Semiconductor Silicon quantum dots (SiQDs) and magnetic nanomaterials have been studied extensively for their variety of applications. We have presented a new method for the preparation of Magnetic Silicon Quantum Dots $\left(\mathrm{Fe}_{3} \mathrm{O}_{4} / \mathrm{SiQDs}\right)$ heterostructure nanocomposites. These nanocomposites are fluorescent, have excellent magnetic properties as well as high photocatalytic activity. Magnetic nanoparticles-semiconductor nanocomposites served as an effective recoverable photocatalyst for melamine degradation. In addition, due to their easy magnetic separation, these nanocomposites showed optimum catalytic activity for 15 cycles of usage.

Keywords: melamine, photocatalysis, heterostructure nanocomposites, silicon quantum, dots, magnetic nanoparticles, recycling.
\end{abstract}

\section{INTRODUCTION}

Melamine (1, 3, 5-triazine-2, 4, 6-triamine, $\left.\mathrm{C}_{3} \mathrm{H}_{6} \mathrm{~N} 6\right)$ is a basic organic compound and has potential applications as polymer resins or as raw material for the chemical industry. Melamine contains s-triazine ring, which is very stable at high temperature and pressure. Hence, the discharge from melamine processing industries to surface water is undesirable and often subjected to restrictions. Various attempts have been made to find methods for removing this melamine from wastewater [1]. However, during degradation at high temperature, biodegradation or photolysis, melamine derivatives and analogues could be released into the environment [2]. The melamine's oxidation using UV-irradiation in $\mathrm{H}_{2} \mathrm{O}_{2}$ presence takes place step by step resulting in ammeline, ammelide, and finally to cyanuric acid. Initially, the toxicity of the photocatalyzed solutions is higher than observed for melamine because of the intermediates engendered during photo-oxidation [3]. Also, UV treatment and oxidants had a profound effect in the toxicity of the by-products. Hence there is a need to find an inert, non-toxic and environmentally stable photocatalyst that could catalyze the melamine degradation without UV and oxidants.

Silicon ( $\mathrm{Si}$ ) quantum dots are powerful singlet-oxygen generators in solution and are effective photosensitizers. Compared to $\mathrm{ZnS}$ or CdSe quantum dots, Si nanostructures are inert, non-toxic, abundant, low-cost and biocompatible. Hence, they are arguably one of the essential materials in the physical, chemical and material science research all over the world. Semiconductor nanoparticles can be utilized as photocatalysts in photodecomposition and

\footnotetext{
${ }^{*}$ Corresponding author. Tel.:+966-17-241-5940; fax: +966-17-241-5941. E-mail address:irahmad@kku.edu.sa (I. Mahmood)
}

organic reactions. Similarly, silicon quantum dots, due to their tunable band-gap emissions from near-infrared to blue have been used as encouraging candidates for dye photodegradation, photoreduction and photocatalyzed selective oxidation [4]. However, recycling of these quantum dots, as a photocatalyst, had been a significant concern. Only a few studies are available on their application as renewable photocatalyst [5]. There are two reasons; first, such nanostructures are usually unstable and easily coalesce with each other due to their large surfaceto-volume ratio; hence it is hard to recover them for multiple uses. Secondly; the process of centrifugation and filtration for recycling nanostructures may cause the loss of them.

Regarding industrial use, attention should also be paid to magnetic nanoparticles because of their low cost and excellent separability. In addition, Semiconductor $\mathrm{Si}$ quantum dots (QDs) and magnetic nanoparticles have been widely used in chromatographic separations, chemical catalysis, optoelectronics, and for bio-sensing and labeling [6]. Based on the photocatalytic activity of SiQD's and their easy recovery using magnetic nanoparticles, this study reports the synthesis and characterization of magnetic silicon quantum nanocomposites. Since, magnetic nanostructure technology to solve environmental pollution has received considerable attention, combining quantum dots with magnetic nanoparticles, could lead to recyclable novel nanocomposites with enhanced photocatalytic activity.

A polyoxometalate (POM) assisted method was used for synthesis of SiQDs: because of the following characteristics: (i) silicon nanoparticles produced are uniform and terminated with $\mathrm{Si}-\mathrm{H}$; (ii) the sizes of SiQDs could be controlled and determined by current density; (iii) the SiQDs have good photoluminescence properties. The 
SiQDs terminated with $\mathrm{Si}-\mathrm{H}$ means that they will possess relatively high chemical reactivity due to a larger electron hole. Moreover, magnetic nanoparticles were prepared via a coprecipitation method, to achieve desired size and magnetization.

Hereby, magnetic nanoparticles-semiconductor nanocomposites may be treated as effective recoverable photocatalysts in organic synthesis or for photodegradation reactions. Although melamine detection using $\mathrm{Si}$ nanostructures is extensively studied using various techniques and various metal nanocatalysts, surprisingly, it has never been tested for its removal from solution using magnetic silicon quantum dots.

\section{EXPERIMENTAL SECTION}

All the chemicals were purchased from SigmaAldrich. Iron oxide nanoparticles were synthesized by the chemical co-precipitation method, as reported earlier [7]. While blue light emitting SiQDs were made by polyoxometalates (POMs) assisted chemical etching method. Briefly, n-type (boron-doped) and p-type (phosphorus-doped) Si chips were first for 5 min cleaned with $20 \%$ hydrofluoric acid (HF), to eliminate any surface oxides and impurities. The electrolyte was prepared by mixing $60 \mathrm{~mL}$ of ethanol/HF (3:2) with a suitable amount (5-10 mL) of $\mathrm{H}_{2} \mathrm{O}_{2}$ (hydrogen peroxide $30 \%$ ) and POM was used as catalyst. POM was used in ethanol solution $\left(10^{-2}-10^{-3} \mathrm{M}\right)$. The composition of hybrid polyoxometalates (HPOM) catalyst was $\mathrm{H}_{2} \mathrm{O}_{2} / \mathrm{POM}: 97: 3$ to $90: 10(\mathrm{~V}: \mathrm{V})$. The electrochemical system consisted of graphite as anode and $\mathrm{Si}$ wafer (n-type or p-type, $8-30 \Omega$ resistivity, about $1 \mathrm{~cm}$ in width, and the total reaction area $0.5-1 \mathrm{~cm}^{2}$ ) as cathode. Wafers of graphite and $\mathrm{Si}$ were placed into electrolyte solution and coupled to the DC power supply. A layer of n-hexane covering the electrolyte solution covered the electrolyte. The current intensity ranged $2-50 \mathrm{~mA} / \mathrm{cm}^{2}$ and the etching process was performed for several hours.

\subsection{Synthesis of magnetic quantum dots nanocomposites}

A simple sono-chemical approach was adopted for the synthesis of magnetic silicon quantum dots nanocomposites. Typically, a mixture of Si-QDs colloidal solution and $\mathrm{Fe}_{3} \mathrm{O}_{4}$ nanoparticles were added to $50 \mathrm{ml}$ n-hexane under inert environment in the presence of nitrogen and ultra-sonication. The sonication of the slurry with the high-intensity ultrasound radiation was carried out for $3 \mathrm{~h}$. The product was then separated by a magnet, washed several times with water and dried at room temperature.

\subsection{Melamine degradation}

To explore the photocatalytic activity of magnetic nanocomposites, melamine was nominated as the test molecule. In a typical experiment, $15 \mathrm{mg}$ of the nanocomposites were added to $30 \mathrm{~mL}$ of $6 \times 10^{-5} \mathrm{M}$ melamine solution. Mixture was allowed to irradiate under visible light and $1 \mathrm{ml}$ of the turbid solution was taken from the reaction mixture, at different time intervals to measure their absorption.

\subsection{Characterization}

The measurements of Transmission electron microscopy (TEM) were carried out using Philips CM20 TEM (operating at voltage $200 \mathrm{kV}$ ). XRD patterns of the samples were taken using Siemens D500 powder diffractometer at room temperature $(\mathrm{Cu} K \alpha, \lambda=1.5418 \AA$ ). FT-IR spectra were measured using a Nicolet Avatar 360 FT-IR spectrophotometer. The colored photoluminescence images were attained by a Leica DMIRB photoluminescence microscope. Atomic Force Microscopy (AFM) images were acquired with a NanoscopeIIIa (Digital Instruments Veeco Metrology Group) using a commercial Etched Silicon Probe (Model TESP) (spring constant of $20-100 \mathrm{~N} / \mathrm{m}$ ). The cantilever was oscillated at or slightly below its resonance frequency with amplitude ranging typically from $20 \mathrm{~nm}$ to $100 \mathrm{~nm}$. The separation dependent interaction force was determined by the bending of the cantilever using optical lever procedure. The laser beam was set fixed on the back of a cantilever and reflected onto the photodetector. The collected signal was processed by a computer that controls the feedback to a piezo-scanner. As a consequence, the surface topography of the sample was obtained by raster-scanning. Absorption spectra were recorded on a UV-VIS Spectrophotometer (Perkin Elmer) at room temperature and under visible light.

\section{RESULTS AND DISCUSSION}

\subsection{Characterization of the $\mathrm{Fe}_{3} \mathrm{O}_{4} / \mathrm{SiQD}$ 's nanocomposites}

Morphology of the as-prepared nanocomposites was first characterized by transmission electron microscopy. From TEM analysis (Fig. 1) of the nanocomposites, it can be seen that these nanostructures have uniform-size and spherical morphology with an average size from $10 \mathrm{~nm}$ to $12 \mathrm{~nm}$, which is analogous to the crystallite size $(10 \pm 5 \mathrm{~nm})$ obtained from the X-ray spectrum by means of the Scherer formula using full width at half-maximum (fwhm) of the (311) reflection.

Semiconductor quantum dots (QDs) are ideal for exceptional fluorescence properties single molecule applications and are also have the feature of well-defined size and detection by AFM [8]. Fig. 2 is a correlated AFMfluorescence image employing QDs as single molecule probes. For typical AFM imaging in tapping mode, we observe lower features than expected for QDs. However, the rough surface indicating small dots can be seen in the $\mathrm{Fe}_{3} \mathrm{O}_{4} / \mathrm{SiQD}$ nanocomposites.

Fluorescence microscopy was performed to investigate the luminescent nature of the composite. It is obvious from the photoluminescence images (Fig. 3) that the magnetic quantum dots nanocomposites emit blue to purple fluorescence, which proved the modification of magnetite samples with purple light emitting Si quantum dots.

The crystalline structure of the photocatalysts was determined by powder X-ray diffraction (XRD). The diffraction patterns and relative intensities of all the peaks matched well with those of magnetite (JCPDS card no. 00-002-1035). The broad XRD peaks clearly showed the crystalline nature of the material (Fig. 4). 


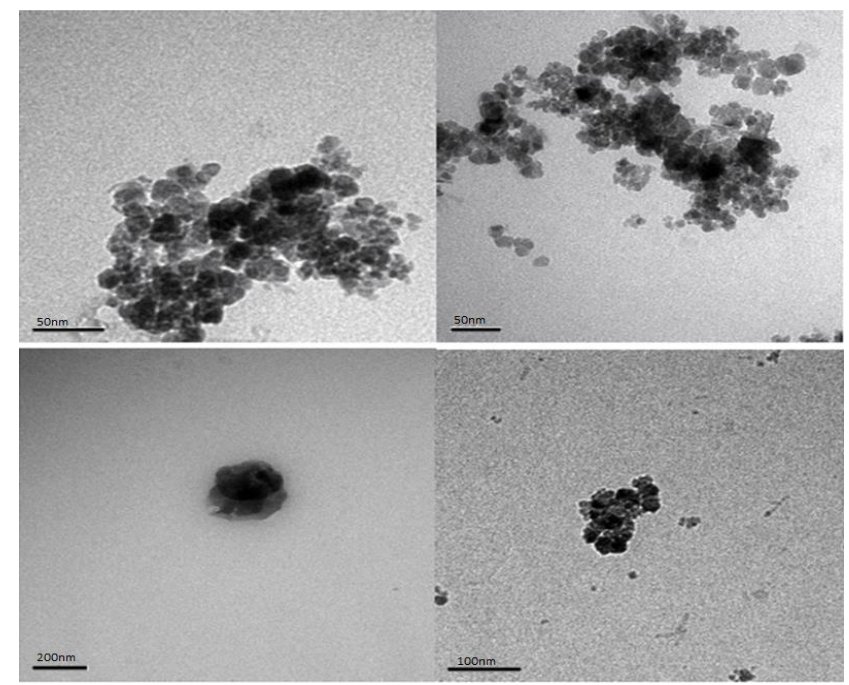

Fig. 1. TEM images of as-prepared $\mathrm{Fe}_{3} \mathrm{O}_{4} / \mathrm{SiQD}$ nanocomposites

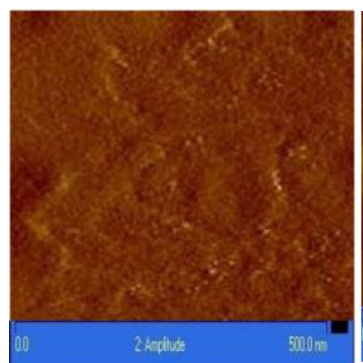

a

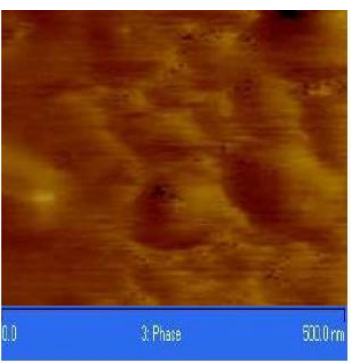

Fig. 2. a-AFM blowups of a region containing SiQDs with overlapping signals in the fluorescence image (scale bar $500 \mathrm{~nm}) ; \mathrm{b}-\mathrm{AFM}$ blowups of a region containing magnetic SiQDs nanocomposites (scale bar $500 \mathrm{~nm}, 45^{\circ}$ )

Magnetic measurements at room temperature showed that there is no significant change in the magnetic moment for $\mathrm{Fe}_{3} \mathrm{O}_{4}$ nanoparticles and $\mathrm{Fe}_{3} \mathrm{O}_{4} / \mathrm{SiQD}$ nanocomposites. Fig. 5 presents the hysteresis loop for $\mathrm{Fe}_{3} \mathrm{O}_{4} / \mathrm{SiQDs}$ nanocomposites.

From VSM measurements, magnetic saturation $\left(M_{\mathrm{s}}\right)$ value of $32 \mathrm{emu} / \mathrm{g}$ was observed for magnetic quantum dots which correspond to strong magnetic responsivity of the nanocomposites.

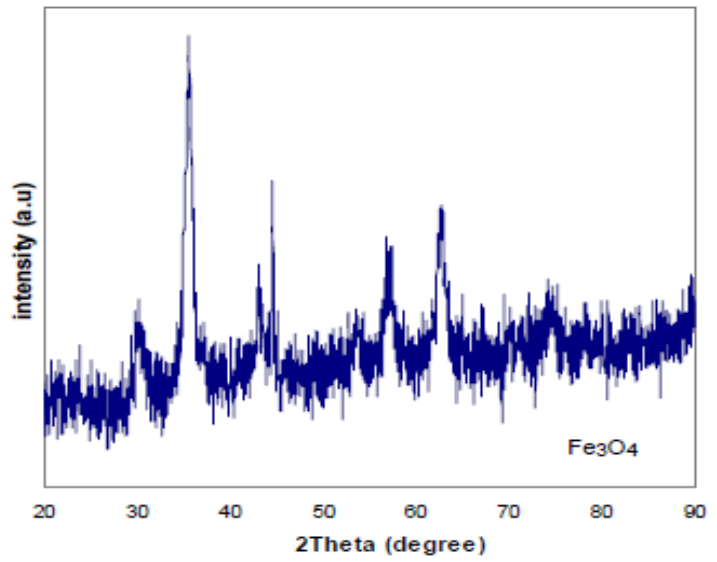

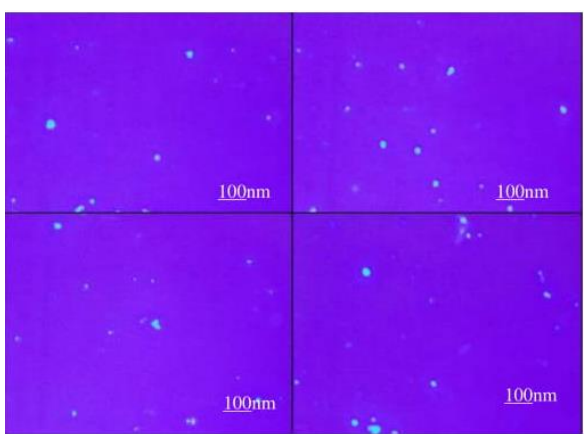

Fig. 3. Florescence images of magnetic Silicon Quantum dots (scale bar; $100 \mathrm{~nm}$ )

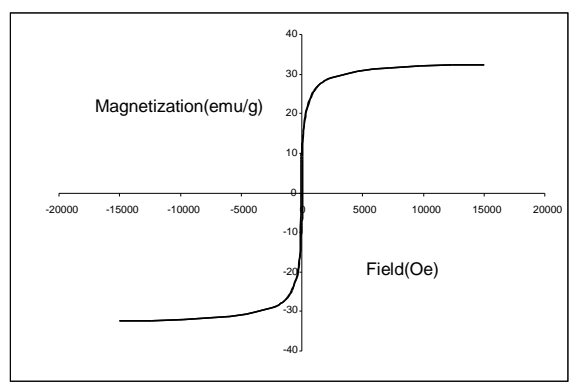

Fig. 5. Magnetic hysteresis loops of $\mathrm{Fe}_{3} \mathrm{O}_{4} / \mathrm{SiQD}$ nanocomposites at room temperature

Fig. 6 presents the FTIR spectra of magnetic nanoparticles, silicon quantum dots and magnetic SiQD nanocomposites in the presence of SiQD's. The characteristic absorption of $\mathrm{Fe}_{3} \mathrm{O}_{4}$ was at $582.5 \mathrm{~cm}^{-1}$ and the peak at $1260 \mathrm{~cm}^{-1}$ corresponds to $\mathrm{O}-\mathrm{H}$ vibrations. The peak at $3412 \mathrm{~cm}^{-1}$ observed in curve (a) relates to the $\mathrm{OH}$ group. One of the important reflections appeared as a sharp decrease in the intensity of the $v(\mathrm{OH})$ band at $3412 \mathrm{~cm}^{-1}$ in the spectrum of the $\mathrm{Fe}_{3} \mathrm{O}_{4} / \mathrm{SiQDs}$ nanocomposites. On the other hand, the suppression of $-\mathrm{OH}$ vibrational modes in the $3000-3700 \mathrm{~cm}^{-1}$ regions has been associated to the proof of host guest interaction as a result of complete water release upon inclusion [9]. The decrease could be inferred as resulting from total deprotonation of the hydroxyl groups of $\mathrm{Fe}_{3} \mathrm{O}_{4}$.

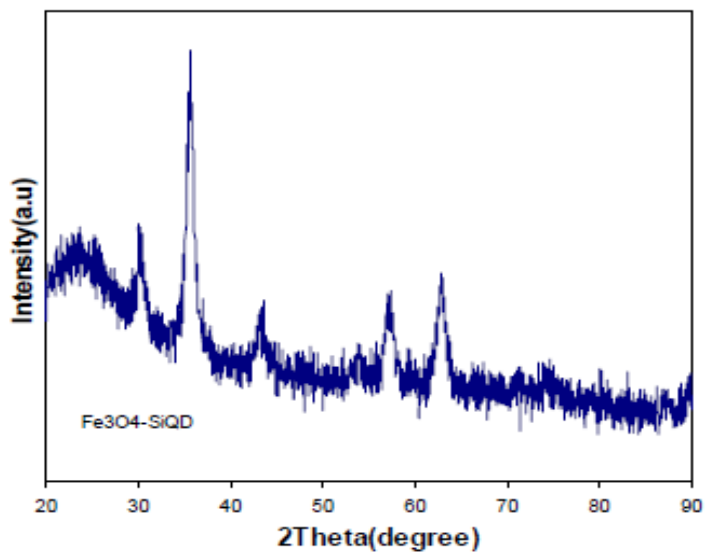

b

Fig. 4. a-XRD patterns of $\mathrm{Fe}_{3} \mathrm{O}_{4}$ nanoparticles; $\mathrm{b}$ - as-prepared $\mathrm{Fe}_{3} \mathrm{O}_{4} / \mathrm{SiQD}$ nanocomposites 

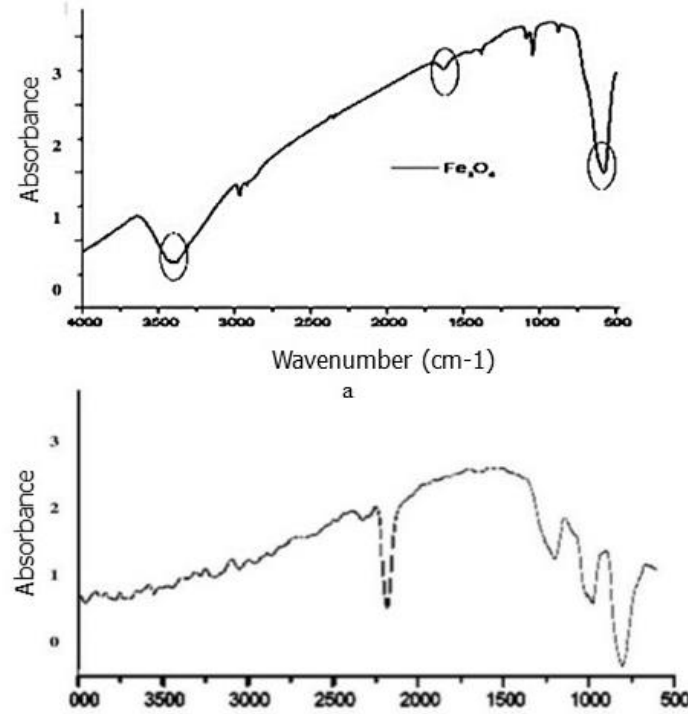

Wavenumber $(\mathrm{cm}-1)$

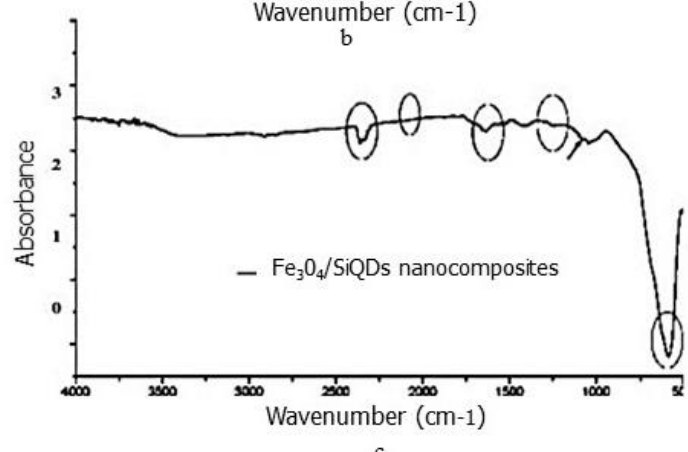

Fig. 6. FTIR spectra: $\mathrm{a}-\mathrm{Fe}_{3} \mathrm{O}_{4} ; \mathrm{b}-$ silicon quantum dots; $\mathrm{c}-\mathrm{Fe}_{3} \mathrm{O}_{4} / \mathrm{SiQD}$ nanocomposites

However, Wenz [9] reported earlier that severe chemical conditions such as high temperature, strong reducing agent $(\mathrm{NaH})$ and absence of water are critical for the deprotonation to occur, which is not the case here. Hence, we can suggest that $-\mathrm{OH}$ groups of magnetic nanoparticles may form hydrogen bonding with SiQD, resulting in $\mathrm{H}-\mathrm{O}-\mathrm{H}$ interactions and thereby reducing the intensity of $\mathrm{OH}$ bands in the spectra. This hypothesis has been reported earlier, published elsewhere [10]. The band at $1044 \mathrm{~cm}^{-1}$ represents $\mathrm{Si}-\mathrm{O}$ bonds (Fig. $6 \mathrm{c}$ ), while band at $1639 \mathrm{~cm}^{-1}$ is due to the stretching and bending vibrations of $\mathrm{Si}-\mathrm{OH}$, which may suggest that $\delta(\mathrm{HOH})$ of two different types of molecules are existing in the solution.

The FTIR spectra shown in Fig. 6 also indicate a change in the bonding configuration for magnetic quantum dot. It has been known that band at $2180-2200 \mathrm{~cm}^{-1}$ represents the $\mathrm{Si}-\mathrm{H}$ stretching mode (Fig. $6 \mathrm{~b}$ ). If the stretching mode at $2180-2200 \mathrm{~cm}^{-1}$ corresponds to $\mathrm{Si}-\mathrm{H}$, the $\mathrm{Si}-\mathrm{Si}$ dimmer will be the largest silicon cluster; hence no composite would be formed. It has been known that the vibrational energy of $\mathrm{Si}-\mathrm{H}$ decreases with an increase in the density of the medium surrounding the $\mathrm{Si}-\mathrm{H}$ bond. Usually, in the presence of a dense material, such as, magnetic nanoparticles, the $\mathrm{Si}-\mathrm{H}$ bond shifts to a lower peak and very weak bond at 2097 appeared. The Si-H vibration mode at $2180-2200 \mathrm{~cm}^{-1}$ can also be explained by the number of hydrogen bonds in $\mathrm{Si}-\mathrm{H}$. The very weak absorption at $1086 \mathrm{~cm}^{-1}$, due to $\mathrm{Si}-\mathrm{O}$ stretching, suggests that very little oxygen is present. Therefore, it can be suggested that for $\mathrm{Fe}_{3} \mathrm{O}_{4} / \mathrm{SiQD}$ nanocomposites, $\mathrm{Si}-\mathrm{H}$ bond mainly formed outside the $\mathrm{Si}-\mathrm{QD}$, i.e. with the $\mathrm{OH}$ - group of $\mathrm{Fe}_{3} \mathrm{O}_{4}$ nanoparticles. These results indicate that the nanocomposites contain a very small amount of individual hydrogenated groups and are present mainly as $\mathrm{Fe}_{3} \mathrm{O}_{4} / \mathrm{SiQD}$ nanocomposites.

\subsection{Photocatalytic activity of $\mathrm{Fe}_{3} \mathrm{O}_{4} / \mathrm{SiQD}$ nanocomposites}

The wavelength tunable emission of Si QDs in the visible range depicts that the direct band gap for of blue light emitting SiQDs corresponds well with the spectrum of sunlight [11]. For instance, Zhen-Hui Kang and coworkers have investigated the photocatalytic reduction of Methyl red using SiQD in visible light for $3 \mathrm{~h}$, and the reduction of methyl red was almost complete. However, literature has reported the highly photocatalytic activity of the semiconductor nanostructures only in the first cycle, and little devotion has been paid to the examination of their renewable photocatalytic properties in the following reaction.

We have observed that the recyclable photocatalytic activity was maintained in the following 15 cycles of usage, as the magnetic parts in the nanocomposites supply us simply with separation. Photocatalytic activity was observed using the method described previously by Wan and co-workers [12]. The absorption spectrum of $\mathrm{Fe}_{3} \mathrm{O}_{4} / \mathrm{SiQD}$ nanocomposites showed an obvious absorption shoulder around $470 \mathrm{~nm}$ (Fig. 7).

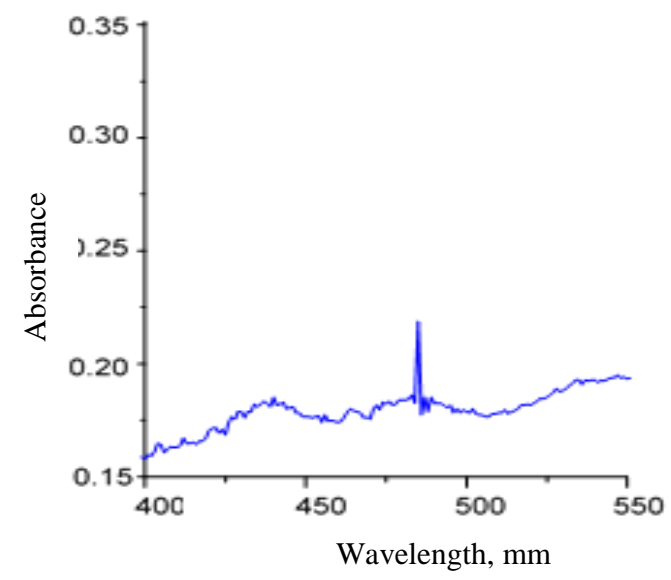

Fig. 7. UV-VIS absorption spectrum peak for $\mathrm{Fe}_{3} \mathrm{O}_{4} / \mathrm{SiQD}$ nanocomposites

Melamine was spectrophotometrically determined at $\lambda \max =655 \mathrm{~nm}$. In addition, the effect of contact time on the absorption of the melamine at different intervals was also observed as shown in Fig. 8. The intensity of the absorption peak decreased gradually and disappeared completely after $20 \mathrm{~min}$.

From Fig. 9, we can see that melamine absorption reached to its maximum. In the presence of $\mathrm{Fe}_{3} \mathrm{O}_{4} / \mathrm{SiQD}$ photocatalyst, melamine concentration decreased rapidly in the solution and degradation was completed within $20 \mathrm{~min}$. However, higher catalytic activity in each cycle of usage is also necessary for process efficiency. 


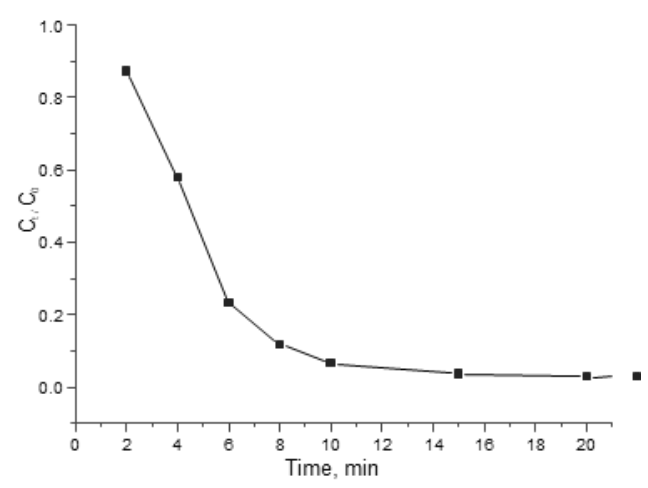

Fig. 8. Photodegradation of melamine $\left(30 \mathrm{~mL}, 6 \times 10^{-5} \mathrm{M}\right)$

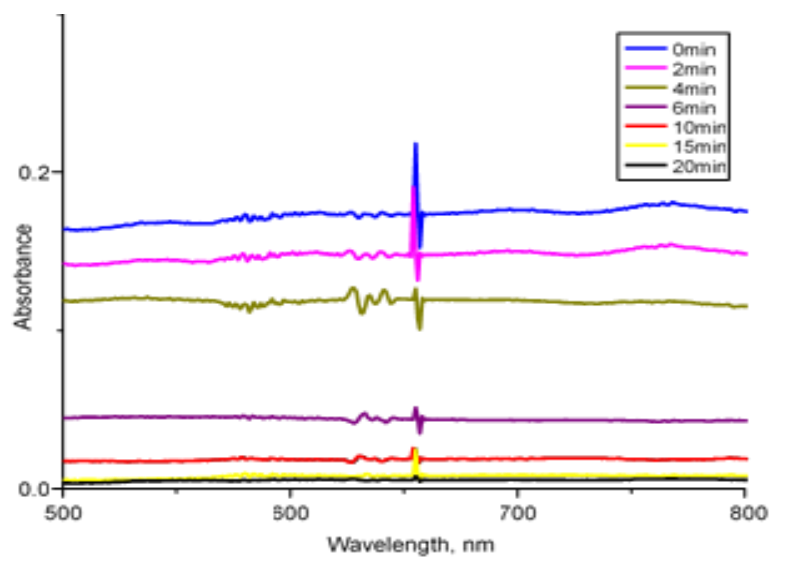

Fig. 9. Absorption spectrum of a solution of Melamine $\left(6.0 \times 10^{-5} \mathrm{M}, 30 \mathrm{~mL}\right)$ in the presence of $\mathrm{Fe} 3 \mathrm{O} 4 / \mathrm{SiQD}$ nanocomposites $(15 \mathrm{mg})$

The photocatalytic activity of the nanocomposites decreased slightly after each cycle. Catalytic activity reached to its maximum within $10 \mathrm{~min}$, and a little change was observed thereafter. This kind of behavior was almost the same, after melamine exposure to the photocatalyst, for all 15 cycles of usage (Fig. 10).

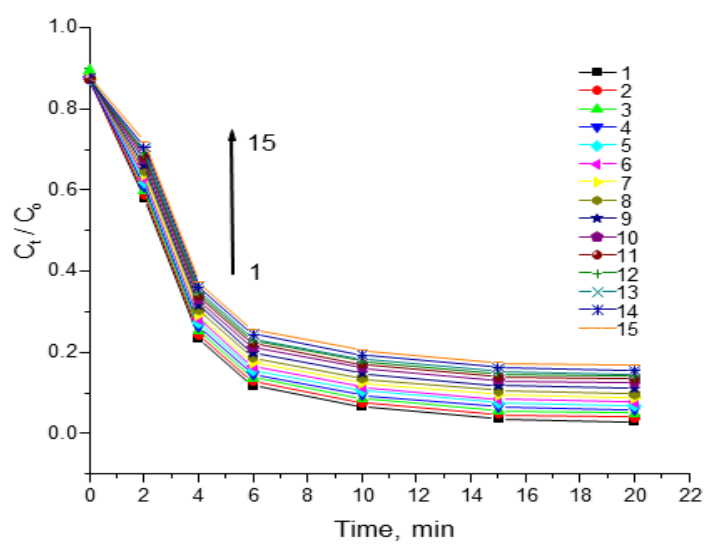

Fig. 10. Behaviours of recycled $\mathrm{Fe}_{3} \mathrm{O}_{4} / \mathrm{SiQD}$ nanocomposites in photodegradation of Melamine $\left(30 \mathrm{~mL}, 6^{\circ} \times{ }^{\circ} 10^{-5} \mathrm{M}\right)$ in different cycles under visible light irradiation

Photocatalytic activity only decreased after 15 cycles of usage, and almost $85 \%$ of melamine degradation was observed in the last cycle. After careful observation of these results, we can conclude that these nanocomposites are an ideal recyclable photocatalyst for melamine degradation in aqueous solution.

\section{CONCLUSIONS}

In conclusion, we have reported the synthesis, characterization and application of an effective and recyclable $\mathrm{Fe}_{3} \mathrm{O}_{4} / \mathrm{SiQD}$ nanocatalyst with high photocatalytic activity towards melamine degradation in aqueous solution. The synthesis method is simple, inexpensive, green, and efficient compared with the former routes utilized to obtain similar nanostructures. In addition, maximum catalytic activity can reach to its maximum within $10 \mathrm{~min}$. Moreover, recyclable photocatalytic activity was maintained following 15 cycles of usage. Thus, as-prepared $\mathrm{Fe}_{3} \mathrm{O}_{4} / \mathrm{SiQD}$ nanocomposites, because of individual catalytic properties of each component, can be used as ideal recyclable photocatalysts in practical applications.

\section{Acknowledgments}

The authors would like to express their gratitude to the deanship of scientific research at King Khalid University; Abha Kingdom of Saudi Arabia for their technical and administrative support.

\section{REFERENCES}

1. Pelizzetti, E., Maurino, V., Minero, C., Carlin, V., Tosato, L.M., Pramauro, E., Zerbinati, O. Photolytic Degradation of Atrazine and other S-Triazine Herbicides Environmental Science and Technology 24 (10) 1990: pp. $1559-1565$.

https://doi.org/10.1021/es00080a016

2. Sancho, J.V., Banezand, M., Grimalt, S., Pozo, Ó.J., Hernández, F. Residue Determination of Cyromazine and Its Metabolite Melamine in Chard Samples by Ion-Pair Liquid Chromatography Coupled To Electrospray Tandem Mass Spectrometry Analytica Chimica Acta 530 (2) 2005: pp. $237-243$. https://doi.org/10.1016/j.aca.2004.09.038

3. Kuo, T.J., Lin, C.N., Kuo, C.L., Huang, M.H. Growth of Ultralong Zno Nanowires on Silicon Substrates by Vapour Transport and Their Use as Recyclable Photocatalysts Chemistry of Materials 19 (21) 2007: pp. 5143-5147. https://doi.org/10.1021/cm071568a

4. Michalet, X., Pinaud, F.F., Bentolila, L.A., Tsay, J.M., Doose, S., Li, J.J., $\quad$ Sundaresan, G., $\quad$ Wu, A.M., Gambhir, S.S., Weiss, S. Quantum Dots for Live Cells, In Vivo Imaging, and Diagnostics Science 307 (5709) 2005: pp. $538-544$

https://doi.org/10.1126/science.1104274

5. Wang, H., Tessmer, I., Croteau, D.L., Erie, D.A., Van Houten, B. Functional Characterization and Atomic Force Microscopy of a DNA Repair Protein Conjugated to a Quantum Dot Nano Letters 8 (6) 2008: pp. 1631-1637. https://doi.org/10.1021/n10803161

6. Murphy, C.J. Optical Sensing with Quantum Dots Analytical Chemistry 74 (19) 2002: pp. 520A-526A. https://doi.org/10.1021/ac022124v

7. Liu, X.Q., Ma, Z.Y., Xing, J.M., Liu, H.Z. Preparation and Characterization of Amino-Silane Modified Superparamagnetic Silica Nanospheres Journal of Magnetism and Magnetic Materials $270(1-2)$ 2004: pp. $1-6$. https://doi.org/10.1016/j.jmmm.2003.07.006

8. Ebenstein, Y., Nahum, E., Banin, U. Tapping Mode Atomic Force Microscopy for Nanoparticles Sizing: Tip- 
Sample Interaction Effects Nano Letters 2 (9) 2002: pp. $945-950$.

https://doi.org/10.1021/n1025673p

9. Wenz, G. Cyclodextrins as Building Blocks for Supramolecular Structures and Functional Units Angewandte Chemie International Edition 33 1994: pp. $803-824$. https://doi.org/10.1002/anie.199408031

10. Bocanegra, A., Novak, M., Mohallem, N.D.S., Sinisterra, R.D. Preparation of Ferrofluid from Cyclodextrin and Magnetite Journal of Magnetism and Magnetic Materials 272-276 (3) 2004: pp. 2395-2397. https://doi.org/10.1016/j.jmmm.2003.12.975

11. Kang, Z.H., Tsang, C.H.A., Ma, D.D.D., Fan, X., Wong, N.B., Lee, S.T. Water-Soluble Silicon Quantum Dots with Wavelength-Tunable Photoluminescence Advanced Materials 21 (6) 2009: pp. 661-664. https://doi.org/10.1002/adma.200801642

12. Hu, J.S., Ren, L.L., Guo, Y.G., Liang, H.P., Cao, A.M., Wan, L.J., Bai, C.L. Mass Production and High Photocatalytic Activity of Zns Nanoporous Nanoparticles Angewandte Chemie International Edition 44 (8) 2005: pp. $1269-1273$.

https://doi.org/10.1002/anie.20046205

(C) Mahmood et al. 2021 Open Access This article is distributed under the terms of the Creative Commons Attribution 4.0 International License (http://creativecommons.org/licenses/by/4.0/), which permits unrestricted use, distribution, and reproduction in any medium, provided you give appropriate credit to the original author(s) and the source, provide a link to the Creative Commons license, and indicate if changes were made. 